\title{
Passivity Analysis of Nonlinear Euler-Bernoulli Beams
}

\author{
MEHRDAD P. FARD $\dagger$ \\ Keywords: Vibration control, distributed parameter systems, passivity
}

The Lagrangian equations for distributed-parameter systems based on Hamilton's principle are developed. These equations are subsequently used to derive nonlinear models for beams. The passivity properties of the flexible mechanical systems based on their distributed-parameter models are then investigated and direct output feedback control laws for control purposes are proposed. Finite gain $L_{2}$ stability and passivity of closed-loop systems are proven. Illustrative cases with simulation of the nonlinear beams and stabilizing feedback control laws are included in the text.

\section{Introduction}

This work is motivated by the industrial interest in stabilizing vibrating slender bodies. This is particularly an area of interest for the offshore engineering community where vibrating slender bodies cause wear and tear due to fatigue. For instance, vortex induced vibrations on marine risers and on floating underwater pipelines are often the dimensioning load with respect to fatigue. Vibrating drill pipes in offshore oil and gas exploration cause reduced drilling productivity and consequently increased costs. Vibrating suspension bridges and energy transmission lines are also of great concern for the industry.

Passivity properties of distributed-parameter systems have been investigated very little in their original form, i.e. partial differential equaions (PDE's). There are few papers which exploit this property of the original models to design control laws. In Matsuno and Murata (1999) the passivity properties of one-link and two-link exible arms, in their original distributed-parameter models, are investigated. In Aamo and Fossen (1999) the passivity of a mooring system, modeled as distributed-parameter system, is proved. Then, it concludes that passive control laws in combination withthe mooring system ensure stability of the total system.

This work establishes the equations of motion and some basic properties of the nonlinear beam equations. The reader is further introduced to the field of automatic control and stabilization of vibrating beams. Illustrative examples are included with simulations. The models are derived from the Lagrangian equations for beams using a distributed parameter approach. The resulting equations are nonlinear. It is proved that the resulting nonlinear systems are passive. The passivity analysis of underlying systems are carried out using the original models. The passivity property is then used to design output feedback control laws for stabilizing vibrating beams. Finite gain $L_{2}$ stability and passivity of the closed loop are proven.

This paper is organized as follows: in Section 2 nonlinear models for beams are derived. Section 3 is devoted to passivity analysis and control design. Numerical

$\dagger$ Norsk Hydro ASA Research Centre, Field Development Dept. Sandsliveien 90 N5020 Bergen-Norway. Tel: +47559968 67, Fax: +47559969 28. Email: Mehrdad.Fard@, hydro.com 
analysis of the underlying systems are conducted in Section 4. The results of numerical simulation are presented in Section 5.

\section{Mathematical Modelling}

For distributed systems, the kinetic and potential energies, in terms of generalized coordinates, can be written as

$$
\begin{aligned}
& V=\int_{0}^{L} \hat{V}\left(\mathbf{q}, \mathbf{q}^{\prime}, \mathbf{q}^{\prime \prime}\right) d x \\
& T=\int_{0}^{L} \hat{T}(\dot{\mathbf{q}}) d x
\end{aligned}
$$

where $\hat{T}$ and $\hat{V}$ are the kinetic and potential energy intensities, respectively. Moreover, the virtual work is simply

$$
\delta W_{n c}=\int_{0}^{L} \mathbf{f}(x, t) \delta \mathbf{q} d x
$$

where $\mathbf{f}(x, t)$ is a vector of the generalized forces corresponding to the generalized coordinate, q. Concentrated forces can be expressed as distributed by means of spatial Dirac delta functions. The extended Hamilton's principle

$$
\int_{t_{1}}^{t_{2}} \delta\left(T-V-W_{n c}\right) d t=0 \quad \delta q_{k}\left(t_{1}\right)=\delta q_{k}\left(t_{2}\right)=0 \quad k=1,2, \ldots, n
$$

requires variation of the Lagrangian $L=T-V$.

$$
\delta L=\int_{0}^{L}\left(\frac{\partial \hat{L}}{\partial \mathbf{q}} \delta \mathbf{q}+\frac{\partial \hat{L}}{\partial \mathbf{q}^{\prime}} \delta \mathbf{q}^{\prime}+\frac{\partial \hat{L}}{\partial \mathbf{q}^{\prime \prime}} \delta \mathbf{q}^{\prime \prime}+\frac{\partial \hat{L}}{\partial \dot{\mathbf{q}}} \delta \dot{\mathbf{q}}\right) d x
$$

The integral can be transformed by using integration by parts with respect to $x$ and $t$. It will be assumed that differentiation and variation are interchangeable. First, the integration is carried out with respect to $t$,

$$
\int_{t_{1}}^{t_{2}} \frac{\partial \hat{L}}{\partial \dot{\mathbf{q}}} \delta \dot{\mathbf{q}} d t=-\int_{t_{1}}^{t_{2}} \frac{\partial}{\partial t}\left(\frac{\partial \hat{L}}{\partial \dot{\mathbf{q}}}\right) \delta \mathbf{q} d t
$$

Note that the fact that $\delta \mathbf{q}$ is zero at $t=t_{1}, t_{2}$ is used. The next step is to carry out integration by parts with respect to $x$, thus

$$
\int_{0}^{L} \frac{\partial \hat{L}}{\partial \mathbf{q}^{\prime}} \delta \mathbf{q}^{\prime} d x=\left.\frac{\partial \hat{L}}{\partial \mathbf{q}^{\prime}} \delta \mathbf{q}\right|_{0} ^{L}-\int_{0}^{L} \frac{\partial}{\partial x}\left(\frac{\partial \hat{L}}{\partial \mathbf{q}^{\prime}}\right) \delta \mathbf{q} d x
$$

Similarly, the next term can be transformed as follows:

$$
\int_{0}^{L} \frac{\partial \hat{L}}{\partial \mathbf{q}^{\prime \prime}} \delta \mathbf{q}^{\prime \prime} d x=\left.\frac{\partial \hat{L}}{\partial \mathbf{q}^{\prime \prime}} \delta \mathbf{q}^{\prime}\right|_{0} ^{L}-\left.\frac{\partial}{\partial x}\left(\frac{\partial \hat{L}}{\partial \mathbf{q}^{\prime \prime}}\right) \delta \mathbf{q}\right|_{0} ^{L}+\int_{0}^{L} \frac{\partial^{2}}{\partial x^{2}}\left(\frac{\partial \hat{L}}{\partial \mathbf{q}^{\prime \prime}}\right) \delta \mathbf{q} d x
$$


Introducing equations (3-8) into (4) results in

$$
\begin{aligned}
\int_{t_{1}}^{t_{2}}\left\{\left[\left.\frac{\partial \hat{L}}{\partial \mathbf{q}^{\prime}} \delta \mathbf{q}\right|_{0} ^{L}+\left.\frac{\partial \hat{L}}{\partial \mathbf{q}^{\prime \prime}} \delta \mathbf{q}^{\prime}\right|_{0} ^{L}-\left.\frac{\partial}{\partial x}\left(\frac{\partial \hat{L}}{\partial \mathbf{q}^{\prime \prime}}\right) \delta \mathbf{q}\right|_{0} ^{L}\right]\right. \\
\left.+\int_{0}^{L}\left[\frac{\partial \hat{L}}{\partial \mathbf{q}^{\prime}}-\frac{\partial}{\partial x}\left(\frac{\partial \hat{L}}{\partial \mathbf{q}^{\prime}}\right)+\frac{\partial^{2}}{\partial x^{2}}\left(\frac{\partial \hat{L}}{\partial \mathbf{q}^{\prime \prime}}\right)-\frac{\partial}{\partial t}\left(\frac{\partial \hat{L}}{\partial \dot{\mathbf{q}}}\right) \delta \mathbf{q}+\mathbf{f}(x, t)\right] \delta \mathbf{q} d x\right\} d t=0
\end{aligned}
$$

At this point, the arbitrariness of the virtual displacement is invoked. If $\delta \mathbf{q}(0, t)=$ $\delta \mathbf{q}(L, t)=0$ and $\delta \dot{\mathbf{q}}(0, t)=\delta \dot{\mathbf{q}}(L, t)=0$, equation (9) is satisfied for all values of $\delta \mathbf{q}$ with $x \in(0, L)$ if and only if

$$
\frac{\partial \hat{L}}{\partial \dot{\mathbf{q}}}-\frac{\partial}{\partial x}\left(\frac{\partial \hat{L}}{\partial \mathbf{q}^{\prime}}\right)+\frac{\partial^{2}}{\partial x^{2}}\left(\frac{\partial \hat{L}}{\partial \mathbf{q}^{\prime \prime}}\right)-\frac{\partial}{\partial t}\left(\frac{\partial \hat{L}}{\partial \dot{\mathbf{q}}}\right)+\mathbf{f}(x, t)=0
$$

for $\forall(x, t) \in(0, L) \times(0, \infty]$. The boundary conditions may be derived from

$$
\left.\frac{\partial \hat{L}}{\partial \mathbf{q}^{\prime}} \delta \mathbf{q}\right|_{0} ^{L}+\frac{\partial \hat{L}}{\partial \mathbf{q}^{\prime \prime}} \delta \mathbf{q}^{L}-\left.\frac{\partial}{\partial x}\left(\frac{\partial \hat{L}}{\partial \mathbf{q}^{\prime \prime}}\right) \delta \mathbf{q}\right|_{0} ^{L}=0
$$

Boundary conditions are obtained by considering that either $\delta \mathbf{q}(0, t)$ or its coefficients are zero and either $\delta \dot{\mathbf{q}}(0, t)$ or its coefficient is zero. Similar statements can be made about the conditions at $x=L$. Equation (10) represents the Lagrangian equation of motion for distributed-parameter system with Lagrangian given by $L=T-V$, where $T$ and $V$ are as in equations (1-2).

It is worth noting that the Lagrange equation, equation (10), was derived for systems with Lagrangian given by cquations (1-2). Possible surices of potential energy at the boundaries, like for instance springs were not considered. In cases where such devices are attached to the boundaries, the potential energy due to these devices can be added to the expression for potential energy equation (1). The inclusion of these terms does not affect the Lagrangian equation but changes the boundary conditions for that particular system.

\subsection{Coupled dynamic system}

Figure 1 shows a beam in bending under the distributed transverse force $f(x, t)$. It is assumed that the beam is pre-tensioned with a constant axial force, $P_{0}$, at its boundary. In addition to this force, the beam is subjected to a time varying axial force which is caused by the elongation of the beam in the longitudinal direction and the bending of the beam in the transverse direction. The significant physical properties of the beam are the flexural rigidity, or bending stiffness, $E I(x)$, the mass per unit length $\rho A(x)$ and the axial stiffness $E A(x)$. These parameters are functions of independent variable $x$. The variables $\eta(x, t)$ and $\mu(x, t)$ denote the transverse and longitudinal displacements respectively, and vary with position and time.

This section presents the derivation of the equations of motion for a modified, nonlinear Euler-Bernoulli beam. The coupling in longitudinal and transverse dynamics are included in the model. It is assumed that the beam is uniform along its length, and accordingly the physical properties of the beam are assumed constant. 


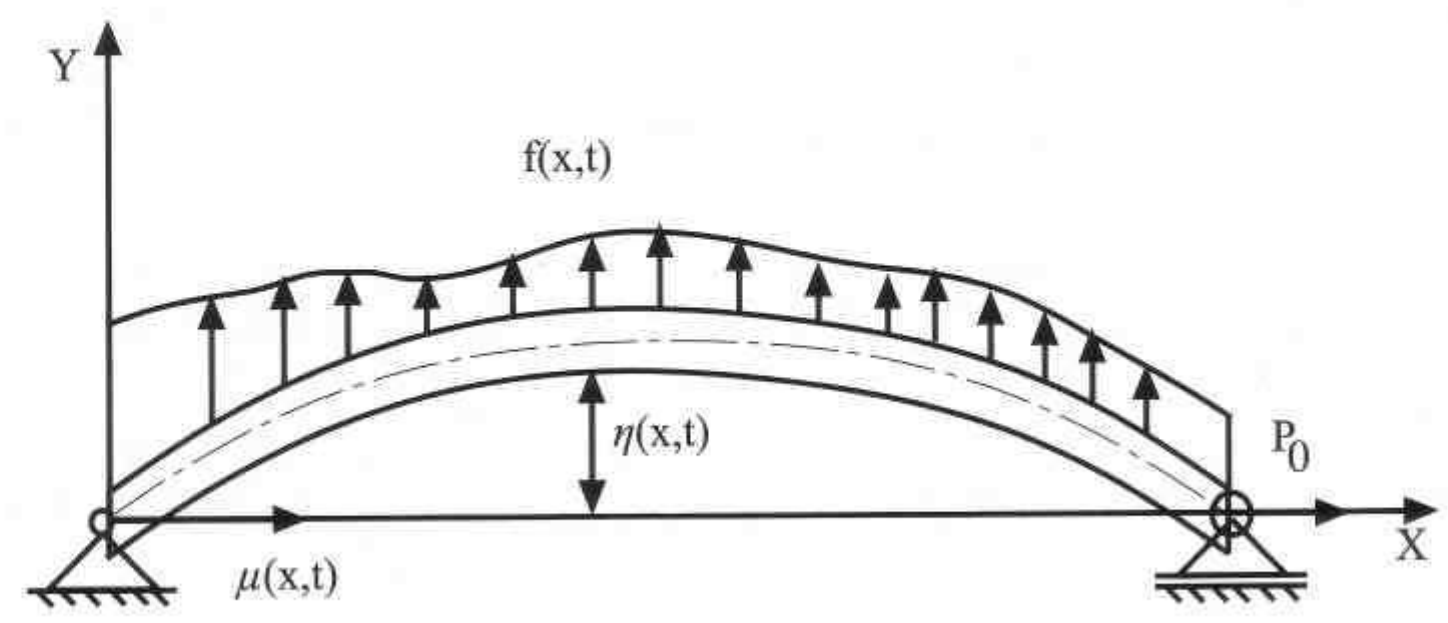

Figure 1. A transversely vibrating beam with axial force.

Henceforth in this paper,for the sake of simplicity and brevity, the notation

$$
(\cdot)_{t t}=\frac{\partial^{2}(\cdot)}{\partial t^{2}},(\cdot)_{x}=\frac{\partial(\cdot)}{\partial x}
$$

and so on will be used. The Lagrangian, $L=T-V$, for the beam configuration shown in Figure 1 is given by

$$
\begin{aligned}
& V(t)=\frac{1}{2} \int_{0}^{L}\left[E \eta_{x x}^{2}+P_{0} \eta_{x}^{2}+E A\left(\mu_{x}+\frac{1}{2} \eta_{x}^{2}\right)^{2}\right] d x \\
& T(t)=\frac{1}{2} \int_{0}^{L} \rho A\left[\eta_{t}^{2}+\mu_{t}^{2}\right] d x
\end{aligned}
$$

where $T(t)$ and $V(t)$ represent the kinetic and potential energy respectively. The expression for the potential energy consists of three parts. The first term is due to bending, the second term is due to the axial force, and the third term is the strain energy of the beam. The generalized coordinate vector $\mathbf{q}$ is defined as $\mathbf{q}^{T}=[\mu(x, t)$, $\eta(x, t)]^{T}$. The generalized force vector is given by

$$
\mathbf{f}(x, t)=[0, f(x, t)]^{T}
$$

Computation of terms in (10) yields

$$
\begin{aligned}
& \frac{\partial}{\partial x}\left(\frac{\partial \hat{L}}{\partial q_{1}^{\prime}}\right)=E A \mu_{x}(x, t)+\frac{1}{2} \frac{\partial}{\partial x}\left(E A \eta_{x}^{2}\right) \\
& \frac{\partial}{\partial t}\left(\frac{\partial \hat{L}}{\partial \dot{q}_{1}}\right)=\rho A \mu_{t t} \\
& \frac{\partial}{\partial x}\left(\frac{\partial \hat{L}}{\partial q_{2}^{\prime}}\right)=P_{0} \eta_{x x}+E A \frac{\partial}{\partial x}\left(\mu_{x} \eta_{x}\right)+\frac{1}{2} E A \frac{\partial}{\partial x}\left(\eta_{x}^{3}\right)
\end{aligned}
$$




$$
\begin{gathered}
\frac{\partial^{2}}{\partial x^{2}}\left(\frac{\partial \hat{L}}{\partial q_{2}^{\prime \prime}}\right)=\frac{\partial^{2}}{\partial x^{2}}\left(\frac{\partial \hat{L}}{\partial \eta_{x x}}\right)=E I \eta_{x x x x} \\
\frac{\partial}{\partial t}\left(\begin{array}{c}
\partial \hat{L} \\
\partial \dot{q}_{2}
\end{array}\right)=\frac{\partial}{\partial t}\left(\frac{\partial \hat{L}}{\partial \eta_{t}}\right)=\rho A \eta_{t t}
\end{gathered}
$$

Substitution of the expressions equations (12-17) into (10), yields

$$
\begin{gathered}
\rho A \eta_{t t}+E I \eta_{x x x x}-P_{0} \frac{\partial^{2} \eta(x, t)}{\partial x^{2}}+E A \frac{\partial}{\partial x}\left(\mu_{x} \eta_{x}\right)-\frac{E A}{2} \frac{\partial}{\partial x}\left(\eta_{x}^{3}\right)-f(x, t)=0 \\
\rho A \mu_{t t}-E A \mu_{x x}-\frac{1}{2} E A \frac{\partial}{\partial x}\left(\eta_{x}^{2}\right)=0
\end{gathered}
$$

The boundary conditions for a simply supported beam are obtained, using equation (11), as

$$
\begin{array}{rlrl}
\frac{1}{2} E A \eta_{x}^{2}+E A \mu_{x}=u_{l}(t), & & \text { at } x=L \\
E I \eta_{x x} & =0, & & \text { at } x=0, L \\
\eta(x, t) & =0, & & \text { at } x=0, L \\
\mu(x, t) & =0, & & \text { at } x=0
\end{array}
$$

where $u_{l}(t)$ represents the control input.

\subsection{Transverse bending of a beam}

In many cases it is desirable, or sufficient, to consider only the transverse dynamic of a beam and neglect the coupling between transverse and longitudinal dynamic. This corresponds to the case where it is assumed that the strain-displacement relation is given by

$$
P(x, t)=P_{0}+\frac{1}{2} E A \eta_{x}^{2}
$$

Consider the Lagrangian

$$
L=\frac{1}{2} \int_{0}^{L}\left[\rho A \eta_{t}^{2}-E I \eta_{x x}^{2}-P_{0} \eta_{x}^{2}-E A \frac{1}{4} \eta_{x}^{4}\right] d x
$$

for $\forall(x, t) \in(0, L) \times[0, \infty)$. Following the same line of reasoning as in Section 2.1, one can obtain the equation of motion

$$
\rho A \eta_{t t}+E I \eta_{x x x x}-P_{0} \eta_{x x}-\frac{E A}{2} \frac{\partial}{\partial x}\left(\eta_{x}^{3}\right)-f(x, t)=0
$$

With reference to equation (11) the boundary conditions are obtained as

$$
-E I \eta_{x x x}+P_{0} \eta_{x}+\frac{E A}{2} \eta_{x}^{3}=u_{T}(t), \quad \text { at } x=L
$$




$$
\begin{aligned}
& E I \eta_{x x}=0, \quad \text { at } x=0, L \\
& \eta(x, t)=0, \quad \text { at } x=0
\end{aligned}
$$

where $u_{T}(t)$ is the boundary control input. The boundary control in equation (25) represents the balance of shear force at the boundary.

\subsection{Transversely vibrating beam with control mechanism}

In the previous section there was no active control mechanism for the system. Consider a control mechanism, which consists of a mass-damper-spring (MDS) system that is attached to the boundary of the beam as shown in Figure 2. This mechanism is composed of a lumped mass $m$, a viscous damper with constant coefficient $d$ and a spring with constant stiffness $k$. The boundary control force $u_{c}(t)$ is applied to this MDS system. The equations of motion of the total system in the domain, $0<x<L$, will be the same as in equation (24). Only the boundary condition in equation (25) will be modified to take into account the MDS system of the control mechanism:

$$
-E I \eta_{x x x}+P_{0} \eta_{x}+\frac{E A}{2} \eta_{x}^{3}+m \eta_{t t}+d \eta_{t}+k_{\eta}=u_{c}(t) \quad \text { at } x=L
$$

The new state vector $v=[\eta(x, t) \eta(L, t)]^{\mathrm{T}}$ is now introduced. After introduction of damping into equation (24), the governing equations can be written as

$$
\mathbf{M} \ddot{v}+\mathbf{D} \dot{v}+\mathscr{L} v+\mathscr{F}=\mathbf{U}
$$

where

$$
\begin{aligned}
& \mathbf{M}=\left[\begin{array}{cc}
\rho A & 0 \\
0 & m
\end{array}\right] \\
& D=\left[\begin{array}{ll}
D & 0 \\
0 & d
\end{array}\right]
\end{aligned}
$$

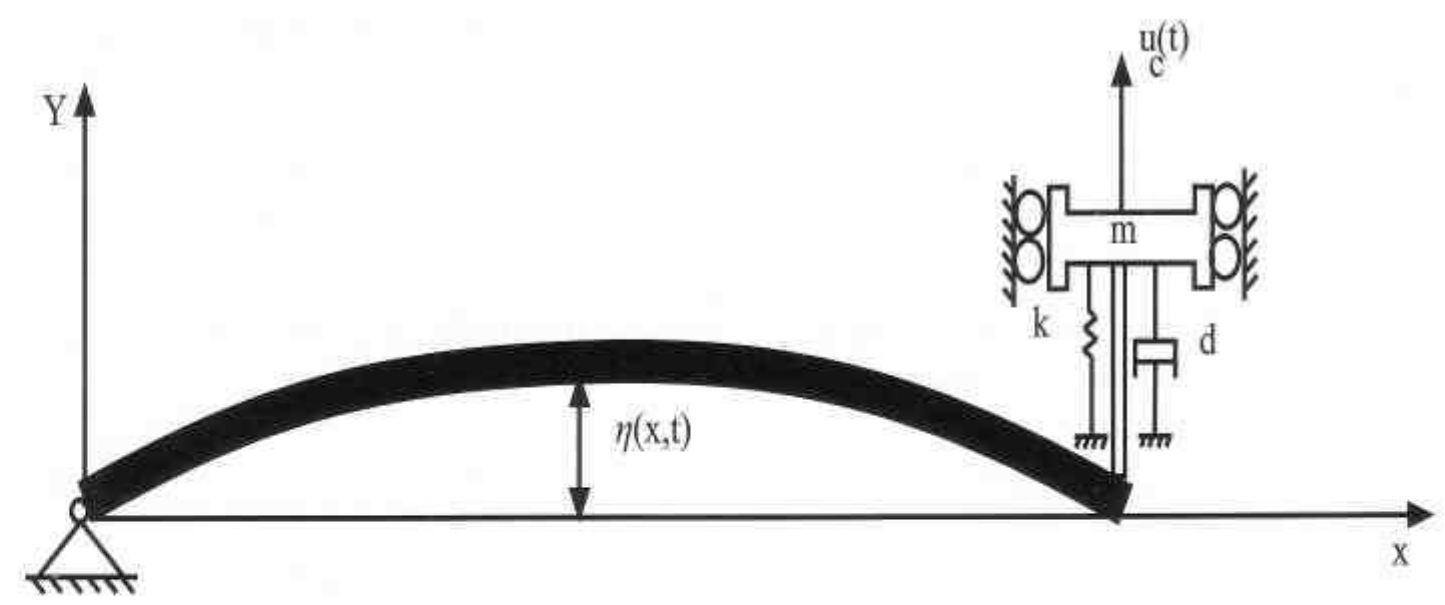

Figure 2. A vibrating beam with a MDS boundary controller. 


$$
\begin{aligned}
& \mathscr{L}=\left[\begin{array}{cc}
\frac{\partial^{2}}{\partial x^{2}} E I \frac{\partial^{2}}{\partial x^{2}}-P_{0} \frac{\partial^{2}}{\partial x^{2}} & 0 \\
0 & -\frac{\partial}{\partial x^{2}} E I \frac{\partial^{2}}{\partial x^{2}}+P_{0} \frac{\partial}{\partial x}+k
\end{array}\right] \\
& \mathscr{F}=\left[\begin{array}{c}
-\frac{1}{2} \frac{\partial}{\partial x}\left(E A \eta_{x}^{3}\right) \\
\frac{1}{2} E A \eta_{x}^{3}
\end{array}\right] \\
& \mathbf{u}=\left[\begin{array}{c}
0 \\
u_{c}(t)
\end{array}\right]
\end{aligned}
$$

\section{Passivity Analysis}

\subsection{Passivity of coupled system}

Proposition 1. The system consisting of equations (18-19) with the boundary conditions (equations (20-23)) is lossless passive when the output $y_{1}(t)$ is given as

$$
y_{1}(t)=\mu_{t}(L, t)
$$

Proof: Consider the storage function

$$
\begin{aligned}
V(t)= & \frac{\rho A}{2} \int_{0}^{L} \eta_{t}^{2}(x, t) d x+\frac{\rho A}{2} \int_{0}^{L} \mu_{t}^{2} d x+\frac{P_{0}}{2} \int_{0}^{L} \eta_{x}^{2}(x, t) d x \\
& +\frac{E I}{2} \int_{0}^{L} \eta_{x x}^{2}(x, t) d x+\frac{E A}{2} \int_{0}^{L}\left(\mu_{x}(x, t)+\frac{1}{2} \eta_{x}^{2}(x, t)\right)^{2} d x .
\end{aligned}
$$

Differentiation of $V(t)$ with respect to time, using the results of Lemma 1 yields

$$
\begin{aligned}
\dot{V}(t)= & \left.E I \eta_{x x x} \eta_{t}\right|_{0} ^{L}+\left.E I \eta_{x x} \eta_{t x}\right|_{0} ^{L}+\left.E A \eta_{x}^{3} \eta_{t}\right|_{0} ^{L}+\left.E A \mu_{x} \mu_{t}\right|_{0} ^{L} \\
& +\left.P_{0} \eta_{x} \eta_{t}\right|_{0} ^{L}+\left.E A \eta_{x} \mu_{x} \eta_{t}\right|_{0} ^{L}+\left.E A \frac{1}{2} \eta_{x}^{2} \mu_{t}\right|_{0} ^{L} \\
\dot{V}(t)= & \left(E A \mu_{x}(L, t)+E A \frac{1}{2} \eta_{x}^{2}(L, t)\right) \mu_{t}(L, t) .
\end{aligned}
$$

Hence, using boundary condition equation (21) and $y_{1}(t)=\mu_{t}(L, t)$ yields $\dot{V}(t)=$ $u_{1}(t) y_{1}(t)$, which completes the proof. 


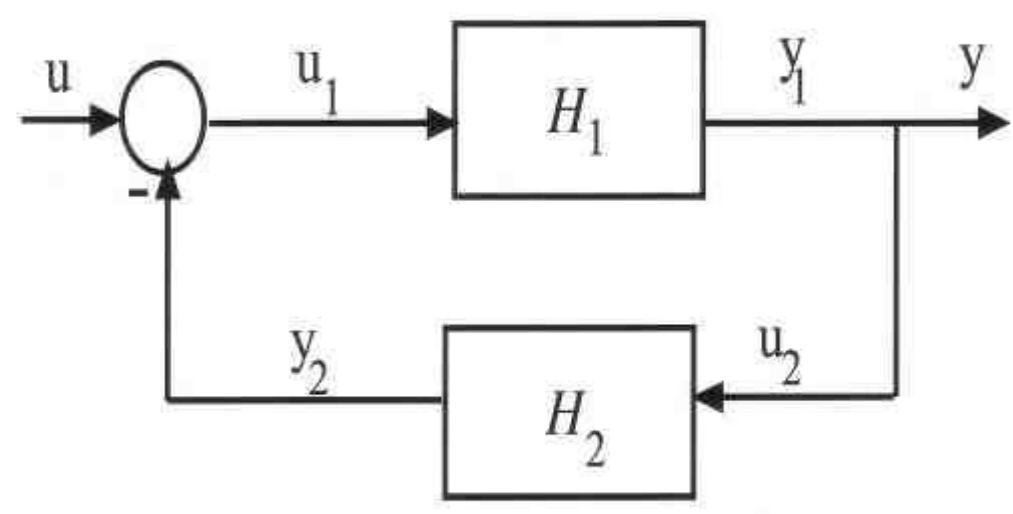

Figure 3. The closed-loop system.

Theorem 1. Consider the feedback system in Figure 3, where $\mathscr{H}_{1}$ is defined by equations (18-19) and (20-23). Let the controller output, $y_{2}(t)$ be

$$
y_{2}(t)=K u_{2}(t)=K \mu_{t}(L, t)
$$

where $K>0$. Then the closed-loop system $\Sigma_{\mathscr{H}_{1}, \mathscr{H}_{2}}$ consisting of equations (18-19) and equations (20-23) with control law eqaution (31) is $\mathscr{L}_{2}$-stable.

Proof: Control law equation (31) is output and input strictly passive according to Definition 10.6 in Khalil (1996), since

$$
u_{2}(t) y_{2}(t)=\frac{K}{2} u_{2}^{2}(t)+\frac{1}{2 K} y_{2}^{2}(t)
$$

According to Theorem 10.6 in Khalil (1996) the closed-loop input-output map $u \rightarrow \mathrm{y}$ is $\mathscr{L}_{2}$-stable.

\subsection{Passivity of transversely vibrating beam}

Proposition 2. The system equation (24) with boundary conditions, equations $25-$ 27 , is lossless passive, when the output $y_{1}(t)$ is given as

$$
y_{1}(t)=\eta_{t}(L, t)
$$

Proof: Consider the storage function

$$
\begin{aligned}
V(t)= & \frac{\rho A}{2} \int_{0}^{L} \eta_{t}^{2}(x, t) d x+\frac{P_{0}}{2} \int_{0}^{L} \eta_{x}^{2}(x, t) d x \\
& +\frac{E A}{8} \int_{0}^{L} \eta_{x}^{4}(x, t) d x+\frac{E I}{2} \int_{0}^{L} \eta_{x x}^{2}(x, t) d x
\end{aligned}
$$

The derivative of $V(t)$ with respect to time, using the results of Lemma 1, yields

$$
\dot{V}(t)=\left(-E I \eta_{x x x}(L, t)+\frac{E A}{2} \eta_{x}^{3}(L, t)+P_{0} \eta_{x}(L, t)\right) \eta_{t}(L, t)
$$

Hence, using the boundary condition equation (25) and $y_{1}(t)=\eta_{t}(L, t)$ yields $\dot{V}(t)=u_{1}(t) y_{1}(t)$ which completes the proof. 
Theorem 2. Consider the feedback system in Figure 3 , where $\mathscr{H}_{1}$ is defined by equation (24) and (25-27). Let the controller output $y_{2}(t)$ be

$$
y_{2}(t)=K u_{2}(t)=K \eta_{t}(L, t)
$$

where $K>0$. Then the closed-loop system $\Sigma_{\mathscr{H}_{1}, \mathscr{H}_{2}}$ consisting of equation (24) and (25-27) with control law equation (33) is $\mathscr{L}_{2}$-stable.

Proof: Control law equation (33) is output and input strictly passive according to Definition 10.6 in Khalil (1996), since

$$
u_{2}(t) y_{2}(t)=\frac{K}{2} u_{2}^{2}(t)+\frac{1}{2 K} y_{2}^{2}(t)
$$

According to Theorem 10.6 in Khalil (1996) the closed-loop input-output map $u \rightarrow y$ is $\mathscr{L}_{2}$-stable.

\subsection{Passivity of transversely vibrating beam with control mechanism}

Proposition 3. The system equation (29) is output strictly passive, when the output $y(t)$ is given by

$$
y(t)=\eta_{t}(L, t)
$$

Proof: Consider the storage function

$$
\begin{aligned}
V(t)= & \frac{\rho A}{2} \int_{0}^{L} \eta_{t}^{2}(x, t) d x+\frac{P_{0}}{2} \int_{0}^{L} \eta_{x}^{2}(x, t) d x+\frac{E A}{8} \int_{0}^{L} \eta_{x}^{4}(x, t) d x \\
& +\frac{E I}{2} \int_{0}^{L} \eta_{x x}^{2}(x, t) d x+\frac{1}{2} m \eta_{t}^{2}(L, t)+\frac{1}{2} k \eta^{2}(L, t) .
\end{aligned}
$$

The derivative of $V(t)$ with respect to time, using the results of the Lemma 1, yields

$$
\begin{aligned}
\dot{V}(t)= & \left(-E I \eta_{x x x}(L, t)+\frac{E A}{2} \eta_{x}^{3}(L, t)+P_{0} \eta_{x}(L, t)\right. \\
& \left.+m \eta_{u}(L, t) \eta t(L, t)+k \eta(L, t)\right) \eta_{t}(L, t)
\end{aligned}
$$

Using the boundary condition equation (28) and $y(t)=\eta_{t}(L, t)$ yields

$$
u_{c}(t) y(t)=\dot{V}(t)+d y^{2}(t)
$$

and, hence, the proof is completed. 


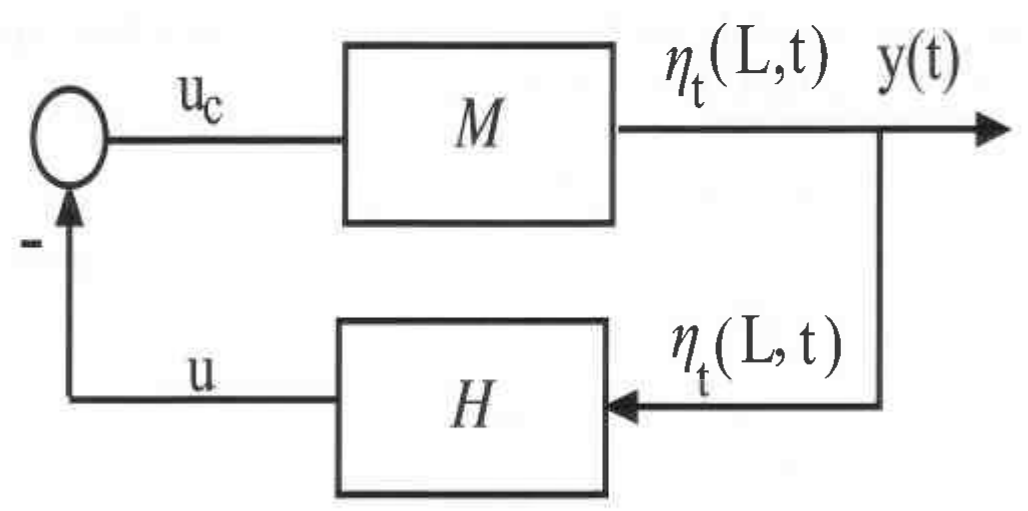

Figure 4. The closed-loop system.

Theorem 3. Consider the closed-loop system $\Sigma_{\mathscr{M}, \mathscr{H}}$ in Figure 4, where

$$
\begin{gathered}
\mathscr{M}: u_{c}(t) \mapsto \eta_{t}(L, t) \\
\mathscr{H}: \eta_{t}(L, t) \mapsto u(t)
\end{gathered}
$$

Let the controller output $u(t)$ be

$$
u(t)=K \eta_{t}(L, t)
$$

where $K>0$. Then, the closed-loop system $\Sigma_{\mathscr{M}, \mathscr{H}}$ consisting of model equation (29) and control law equation (36) is $\mathscr{L}_{2}$-stable.

Proof: The control law equation (36) is output strictly passive according to the Definition 10.6 in Khalil (1996), since

$$
u(t) \eta_{t}(L, t)=K \eta_{t}^{2}(L, t)=\frac{1}{K} u^{2}(t)
$$

According to the passivity theorem, Theorem 2.2.6 in Van der Shaft (1996), $\Sigma_{\mathscr{M}, \mathscr{H}}$ is $\mathscr{L}_{2}$-stable.

\section{Numerical Analysis}

At this point it is convenient to transform the equation of motion equation (24), into nondimensional form. For this purpose the following nondimensional variables (denoted with asterisks) are introduced:

$$
\begin{aligned}
\eta^{*} & =\frac{\eta}{L} \Rightarrow \eta=\eta^{*} L \\
\mu^{*} & =\frac{\mu}{L} \Rightarrow \mu=\mu^{*} L \\
x^{*} & =\frac{x}{L} \Rightarrow x=x^{*} L \\
\tau & =\frac{1}{L} \sqrt{\frac{E}{\rho}} t \Rightarrow t=\sqrt{\frac{\rho}{E}} L \tau
\end{aligned}
$$


where $\tau$ is nondimensional time. Substituting these dimensionless quantities into equation (24), dropping the asterisks, results in:

$$
\eta_{x t}=\alpha \eta_{x x x x}-\beta \eta_{x x}-\frac{3}{2} \eta_{x}^{2} \eta_{x x}=0
$$

where

$$
\begin{gathered}
\alpha=\frac{I}{A L^{2}} \\
\beta=\frac{P_{0}}{E A} .
\end{gathered}
$$

Boundary conditions equations (25-27) can also be transformed into the nondimensional form as

$$
\begin{aligned}
\eta_{x x}(0, \tau) & =\eta_{x x}(1, \tau)=0 \\
\eta(0, \tau) & =0 \\
\bar{u}(\tau) & =-\eta_{x x x}(1, \tau)+\frac{\beta}{\alpha} \eta_{x}(1, \tau)+\frac{1}{2 \alpha} \eta_{x}^{3}(1, \tau)
\end{aligned}
$$

where $\bar{u}(\tau)=u(t)\left(L_{2} / E I\right)$. Equations (37-39) are also valid for the system described in Section 2.3. The boundary condition of equation (28) can be represented in the nondimensional form as

$$
\begin{aligned}
\bar{u}(\tau) & =-\eta_{x x x}(1, \tau)+\frac{\beta}{\alpha} \eta_{x}(1, \tau)+\frac{1}{2 \alpha} \eta_{x}^{3}(1, \tau) \\
& +\frac{L}{\rho I} \eta_{\tau \tau}(1, \tau)+d \sqrt{\frac{E}{\rho} \frac{L^{2}}{E I}} \eta_{\tau}(1, \tau)+k \frac{L^{3}}{E I} \eta(1, \tau) .
\end{aligned}
$$

\subsection{Finite difference analysis}

In order to develop a finite difference scheme approximating the non-dimensional continuous system, equations (37-40), it is assumed that the region to be examined is covered by a uniform rectangular grid with sides parallel to the $x$-and $t$-axes, with $h$ and $k$ being the grid spacing in the $x$ - and $t$-directions respectively. Furthermore, it is assumed that the point considered is the point $x=i h$ and $\tau=j k$ of the domain, where $i$ and $j$ are integers and $i=j=0$ are the origin. The value of the function $\eta(.,$. at the point $x=i h$ and $\tau=j k$ are denoted by $\eta_{i j}$. For a general discussion of finite difference methods the reader is referred to Ames (1965), Mitchell and Griffiths (1980), Richtmyer and Mortonon (1967), Smith (1978).

The following finite difference approximations are used:

$$
\begin{aligned}
& \left(\frac{\partial n}{\partial \tau}\right)_{i, j}=\frac{\eta_{i, j}-\eta_{i, j-1}}{k}+O(k) \\
& \left(\frac{\partial^{2} \eta}{\partial \tau^{2}}\right)_{i, j}=\frac{\eta_{i, j+1}-2 \eta_{i, j}+\eta_{i, j-1}}{k^{2}}+O\left(k^{2}\right)
\end{aligned}
$$




$$
\begin{aligned}
& \left(\frac{\partial \eta}{\partial x}\right)_{i, j}=\frac{\eta_{i+1, j}-\eta_{i-1, j}}{2 h}+O\left(h^{2}\right) \\
& \left(\frac{\partial^{2} \eta}{\partial x^{2}}\right)_{i, j}=\frac{\eta_{i+1, j}-2 \eta_{i, j}+\eta_{i-1, j}}{h^{2}}+O\left(h^{2}\right) \\
& \left(\frac{\partial^{3} \eta}{\partial x^{3}}\right)_{i, j}=\frac{\eta_{i+2, j}-2 \eta_{i+1, j}+2 \eta_{i-1, j}-\eta_{i-2, j}}{2 h^{3}}+O\left(h^{3}\right) \\
& \left(\frac{\partial^{4} \eta}{\partial x^{4}}\right)_{i, j}=\frac{\eta_{i+2, j}-4 \eta_{i+1, j}+6 \eta_{i, j}-4 \eta_{i-1, j}+\eta_{i-2, j}}{h^{4}}+O\left(h^{2}\right)
\end{aligned}
$$

Using equations (42-47), the finite difference approximation to equation (37) takes the form

$$
\begin{aligned}
\eta_{i, j+1}= & -\alpha r^{2}\left(\eta_{i+2, j}-4_{\eta_{i+1, j}}+6 \eta_{i, j}-4 \eta_{i-1, j}+\eta_{i-2, j}\right) \\
& +\beta r^{2} h^{2}\left(\eta_{i+1, j}-2 \eta_{i, j}+\eta_{i-1, j}\right) \\
& +\frac{3}{8} r^{2}\left(\eta_{i+1, j}-\eta_{i-1, j}\right)^{2}\left(\eta_{i+1, j}-2 \eta_{i, j}+\eta_{i-1, j}\right) \\
& +2 \eta_{i, j}-\eta_{i, j-1}
\end{aligned}
$$

where $r$, convergence parameter, is defined as $r=\left(k / h^{2}\right)$. Note that equation (48) is an explicit finite difference equationand has a truncation error of $O\left(h^{6}\right)+O\left(k^{2}\right)$.

\subsection{Truncation error}

Truncation error is defined as the amount by which the exact solution $\eta_{i, j}$ of the differential equation does not satisfy the difference equation at the point $(i h, j k)$. Using finite difference approximation, a difference approximation for equation (37) is obtained as

$$
\begin{aligned}
\frac{1}{k^{2}}\left(\bar{\eta}_{i, j+1}+\bar{\eta}_{i, j-1}-2 \bar{\eta}_{i, j}\right)= & -\frac{\alpha}{h^{4}}\left[\bar{\eta}_{i+2, j}+6 \bar{\eta}_{i, j}-4 \bar{\eta}_{i-1, j}-4 \bar{\eta}_{i+1, j}+\bar{\eta}_{i-2, j}\right] \\
+ & \frac{\beta}{h^{2}}\left(\bar{\eta}_{i+1, j}+\bar{\eta}_{i-1, j}-2 \bar{\eta}_{i, j}\right)+\frac{3}{8 h^{4}}\left(\bar{\eta}_{i+1, j}-\bar{\eta}_{i-1, j}\right)^{2} \\
& \left(\bar{\eta}_{i+1, j}+\bar{\eta}_{i-1, j}-2 \bar{\eta}_{i, j}\right)
\end{aligned}
$$

where $\bar{\eta}_{i j}$ is an approximation to $\eta_{i j}$ at the point $(i h, j k)$. Using Talor's expansion up to the 4 th order in equation (49), the exact solution of the partial equation and its derivatives at different points can be obtained. Substituting into equation (49), after some manipulation the following expression is obtained:

$$
\left(\eta_{\tau \tau}\right)_{i, j}=-\alpha\left(\eta_{x x x x}\right)_{i, j}+\beta\left(\eta_{x x}\right)_{i, j}+\frac{3}{2}\left(\eta_{x}\right)_{i, j}^{2}\left(\eta_{x x}\right)_{i, j}+e_{i, j}+\ldots
$$


where the quantity

$$
\begin{aligned}
e_{i j}= & \frac{h^{2}}{8}\left(\eta_{x}\right)_{i, j}^{2}\left(\eta_{x x x x}\right)_{i, j}+\frac{h^{4}}{24}\left(\eta_{x x x}\right)_{i, j}^{2}\left(\eta_{x x}\right)_{i, j} \\
& +\frac{h^{6}}{288}\left(\eta_{x x x}\right)_{i, j}^{2}\left(\eta_{x x x x}\right)_{i, j}+\frac{h^{2}}{2}\left(\eta_{x}\right)_{i, j}\left(\eta_{x x x}\right)_{i, j}\left(\eta_{x x}\right)_{i, j} \\
& +\frac{h_{4}}{24}\left(\eta_{x}\right)_{i, j}\left(\eta_{x x x}\right)_{i, j}\left(\eta_{x x x x}\right)_{i, j}+\frac{\beta h^{2}}{12}\left(\eta_{x x x x}\right)_{i, j} \\
& -\frac{k^{2}}{12}\left(\eta_{\tau \tau \tau \tau}\right)_{i, j}
\end{aligned}
$$

is defined as the principal part of the truncation error.

\subsection{Consistency or compatibility}

It is sometimes possible to approximate a partial differential equation by a finite difference scheme which is stable but has a solution that converges to the solution of a different differential equation as the mesh lengths tend to zero, Smith (1978). A finite difference scheme is said to be consistent if the limiting value of the truncation error is zero as $h \rightarrow 0$ and $k \rightarrow 0$. It is readily seen from equation (51) that the truncation error tends to zero as $h \rightarrow 0$ and $k \rightarrow 0$. It is concluded that the difference equation scheme in equation (48) is consistent.

\section{Numerical simulation}

In this section the control laws proposed in this paper are simulated. The simulation is carried out for a riserof length $1000[\mathrm{~m}]$. Detailed specifications of the riser are given in Table 1 . The length of the riser is divided into $N+1$ nodes, where $N=100$, and the convergence parameter is chosen as $r=0.0001$. Furthermore, it is assumed that the riser is filled with sea water.

Table 1. Parameter and material properties

\begin{tabular}{lc}
\hline Parameter & Value \\
\hline Mass per unit length & $108.1\left[\frac{\mathrm{kg}}{\mathrm{m}}\right]$ \\
Inner diameter & $76.2 \times 10^{-3}[\mathrm{~m}]$ \\
Outer diameter & $152.4 \times 10^{-3}[\mathrm{~m}]$ \\
Sea water density & $1024\left[\frac{\mathrm{kg}}{\mathrm{m}^{3}}\right]$ \\
Length & $1000[\mathrm{~m}]$ \\
E & $2.06 \times 10^{9}\left[\frac{\mathrm{N}}{\mathrm{m}^{2}}\right]$ \\
\hline
\end{tabular}


Two test cases will be presented to illustrate the performance of the controlled system. In the first case, only the first vibration mode is excited, while in the second case several modes of vibration are excited. The riser is initially at the stress free configuration. At the instant $t=0$, the riser is exposed for sea current along its length. At the instant $t=1$, the sea current is switched off and the riser is left to vibrate freely. At the instant $t=8$, the controller is switched on.

\subsection{Transversely vibrating beam with boundary control}

5.1.1. Case 1. The first vibration mode The sea current profile is modeled as a half period of a sine function and excites only the first transverse bending mode of the riser. Figure 5 shows the responses at different nodes of the riser. The total mechanical energy of the system, equation (32), is shown in Figure 6. The spatial integration is approximated by trapezoidal integration scheme. Since the mapping $u_{1} \mapsto y_{1}$ is lossless passive, the energy does not decreases for $t \leqslant 8$. However, due to the control law, the energy of the system decreases after $t=8$.

5.1.2. Case 2. Several vibration modes The sea current profile is modeled as a sum of sine functions with frequencies up to the tenth mode of vibration. Figure 7 shows the responses of the riser at different nodes. A comparison between control forces for both case studies is shown in Figure 8.

\subsection{Transversely vibrating beam with $M D S$ control mechanism}

The same scenario as in Section 5.1 , with a sea current velocity of $2.9 \mathrm{~m} / \mathrm{s}$ is simulated for this system.
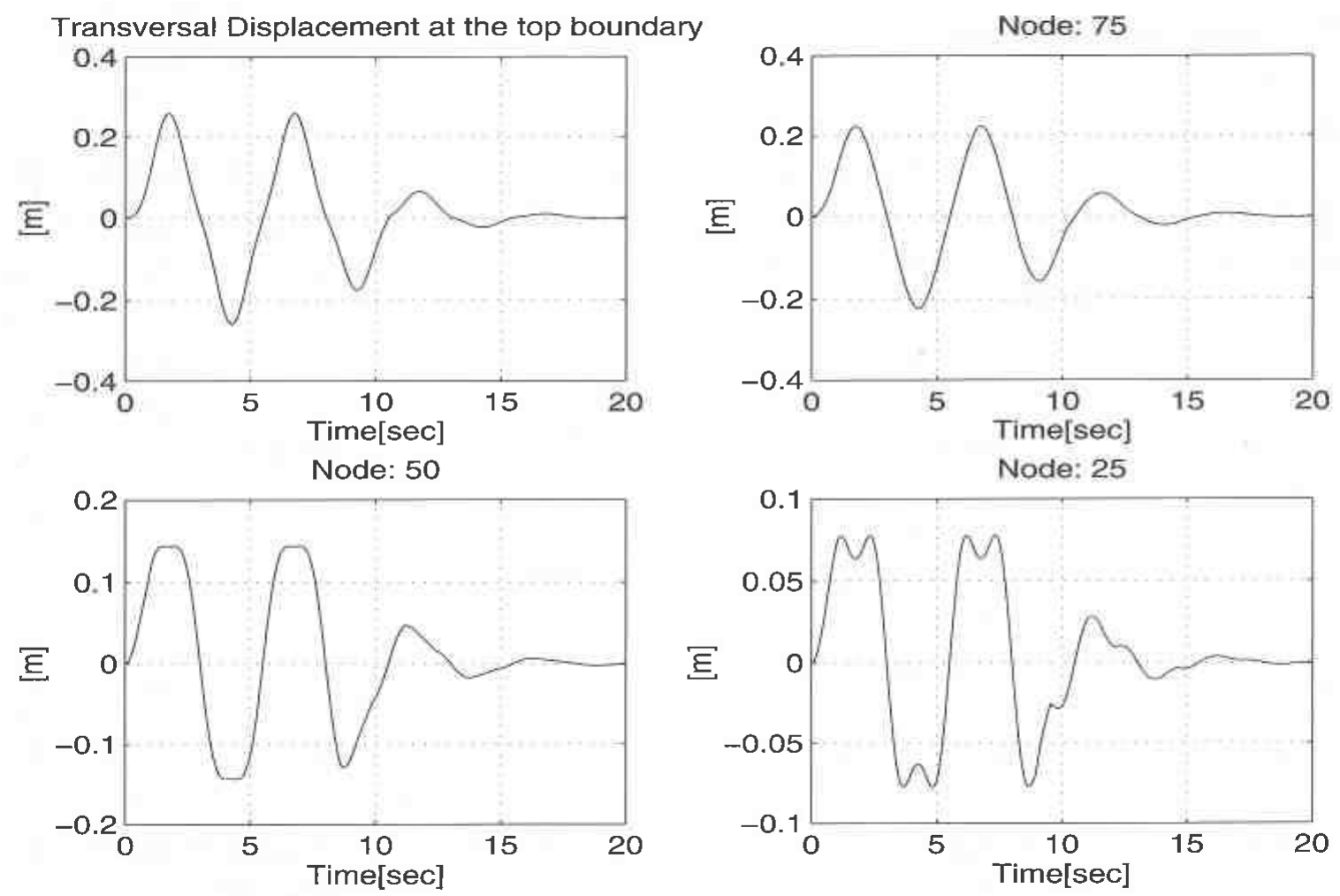

Figure 5. Transverse responses at different nodes, when only the first mode of vibration is excited. 


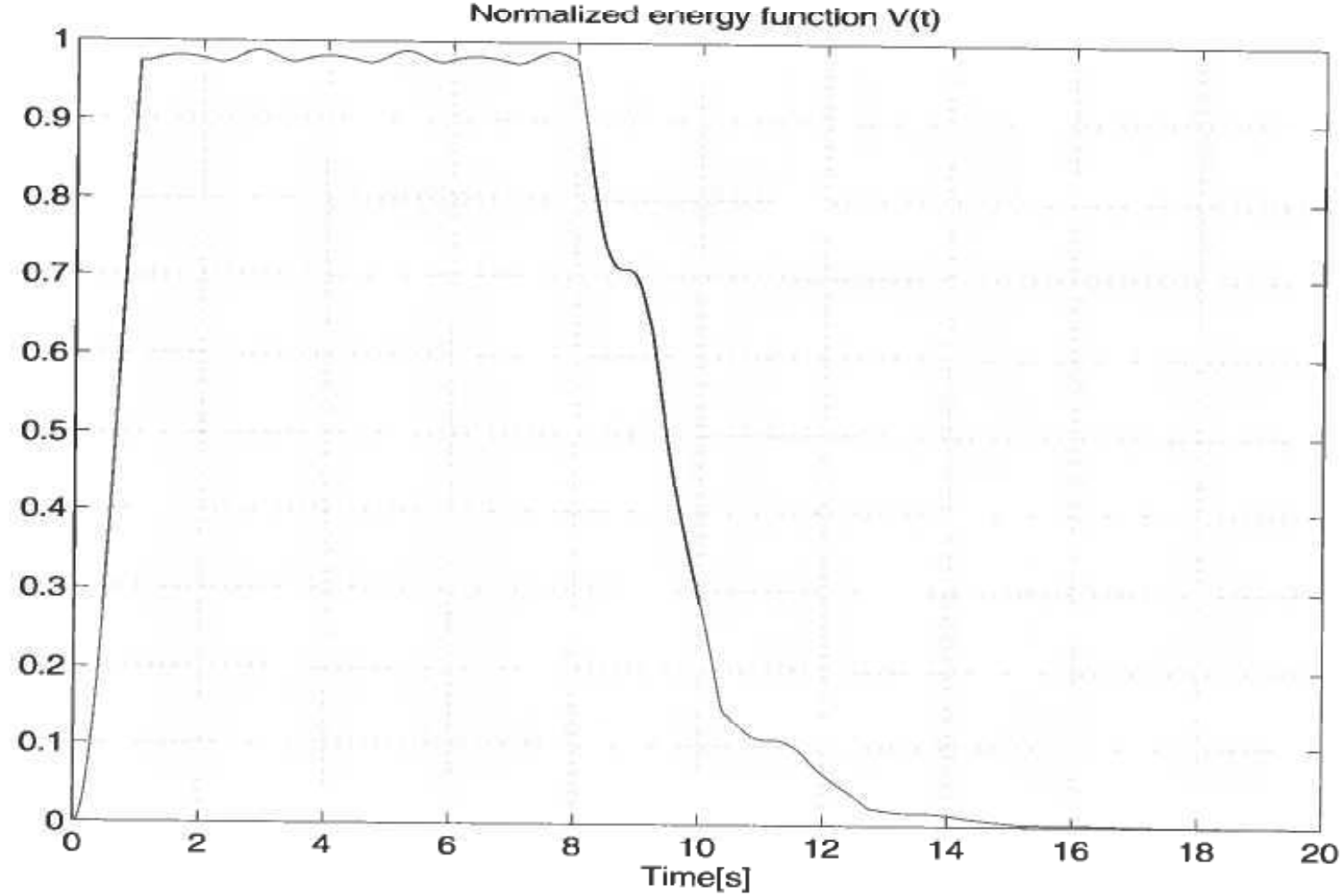

Figure 6. Mechanical energy content of the system.
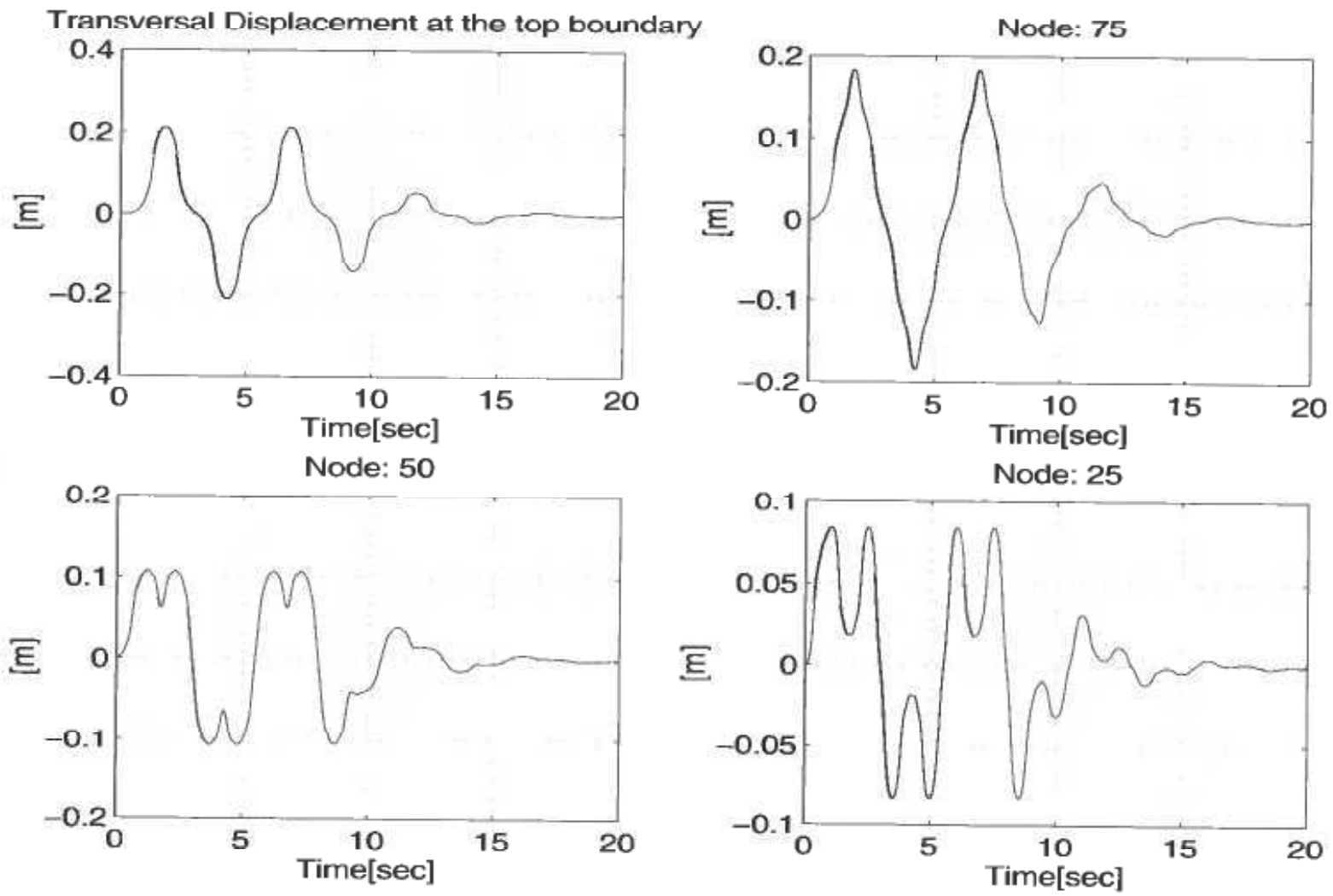

Figure 7. Transverse displacement at different nodes, when several modes of vibration are excited. 


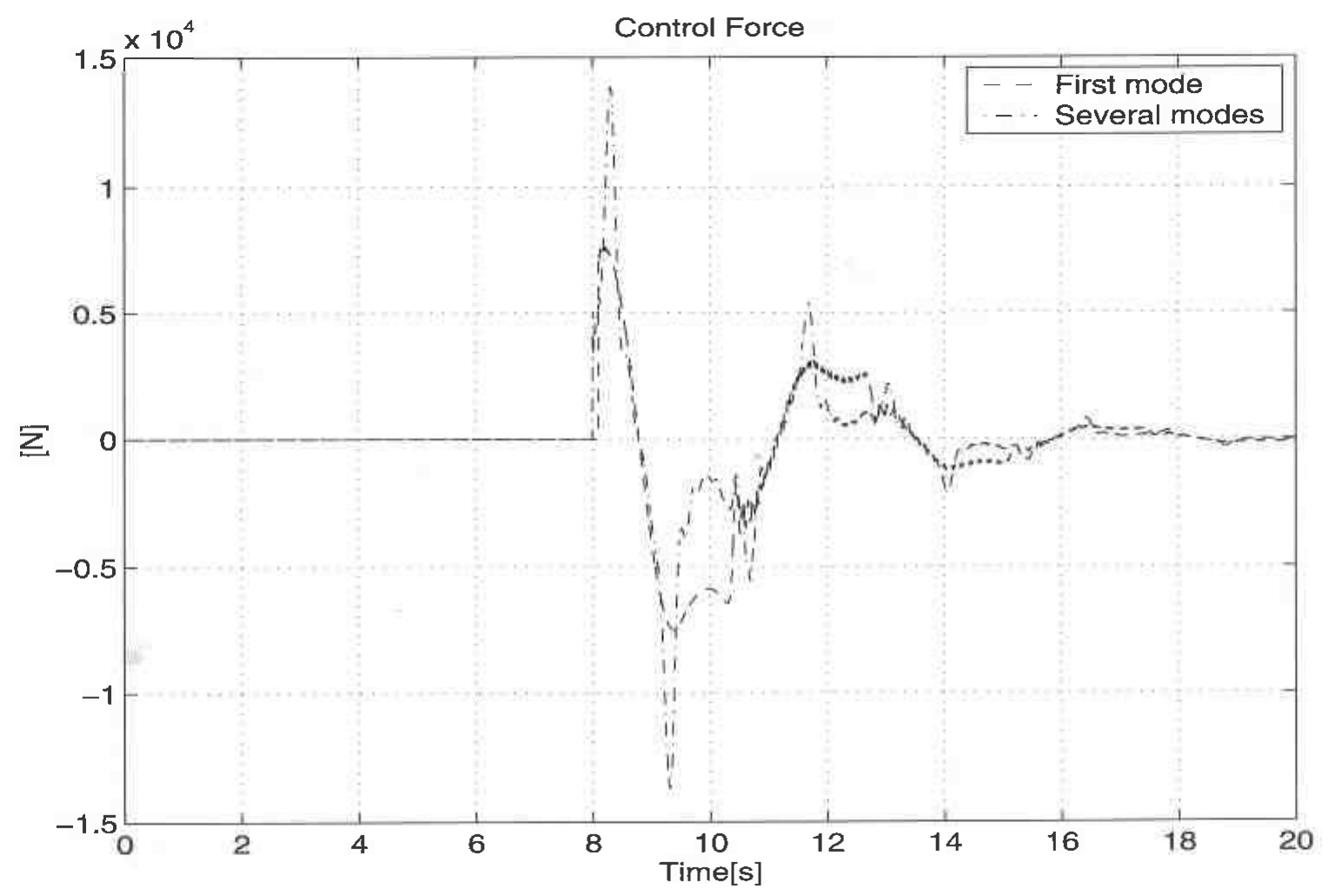

Figure 8. Comparison between control forces.

5.2.1. Case 1. First vibration mode Figure 9 shows the comparison between controlled and uncontrolled responses of the riser when only the first mode of vibration is excited. The total mechanical energy of the system, equation (34), is shown in Figure 10. Since the open-loop system is strictly output passive the energy of the open-loop system decays. The rate of decay increases after the controller is switched on at $t=8$.

5.2.2. Case 2. Several vibration modes Figure 11 shows the transverse responses of controlled and uncontrolled system at different nodes. Acomparison between control forces for both cases is shown in Figure 12.

\section{Conclusion}

Hamilton's principle has been used to derive the Lagrangian equations for distributed-parameter systems. Nonlinear models for Euler-Bernoulli beams are derived and their passivity properties are investigated. Linear output feedback control laws which guarantee finite gain $L_{2}$ stability of the closed-loop systems have been proposed. It is worth noting that any passive control law in combination with the underlying systems may be selected. The passivity of the closed-loop system using Theorem 2.2.3 in Van der Schaft (1996) is still preserved. The finite gain $L_{2}$ stability is also proved, which is more stringent than passivity. In addition to the aforementioned reason, simplicity of proportional controller makes it more attractive, from the implementation point of view. 

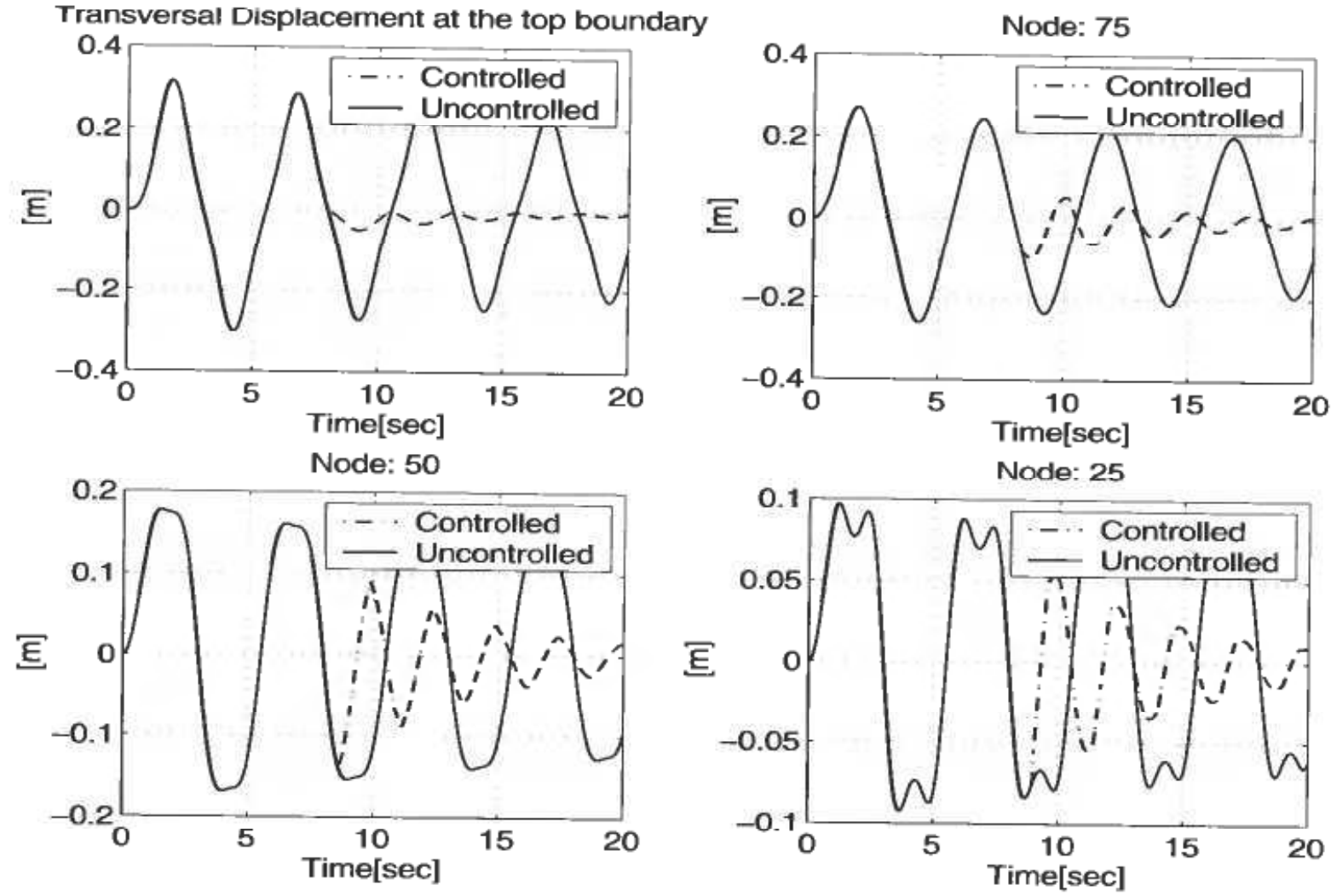

Figure 9. Comparison between controlled and uncontrolled responses of the riser, when only the first mode of vibration is excited.

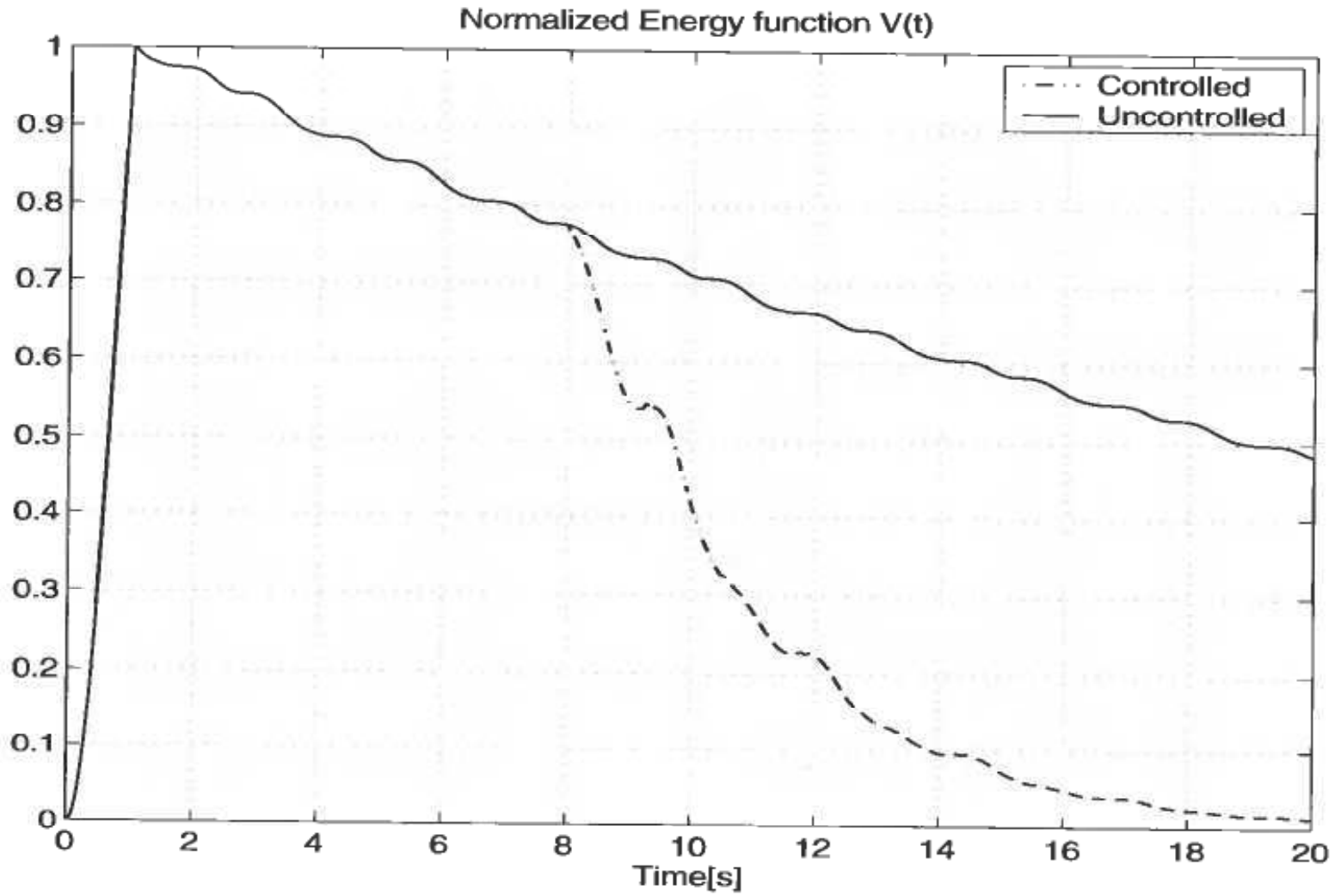

Figure 10. Mechanical energy content of the system with MDS mechanism. 

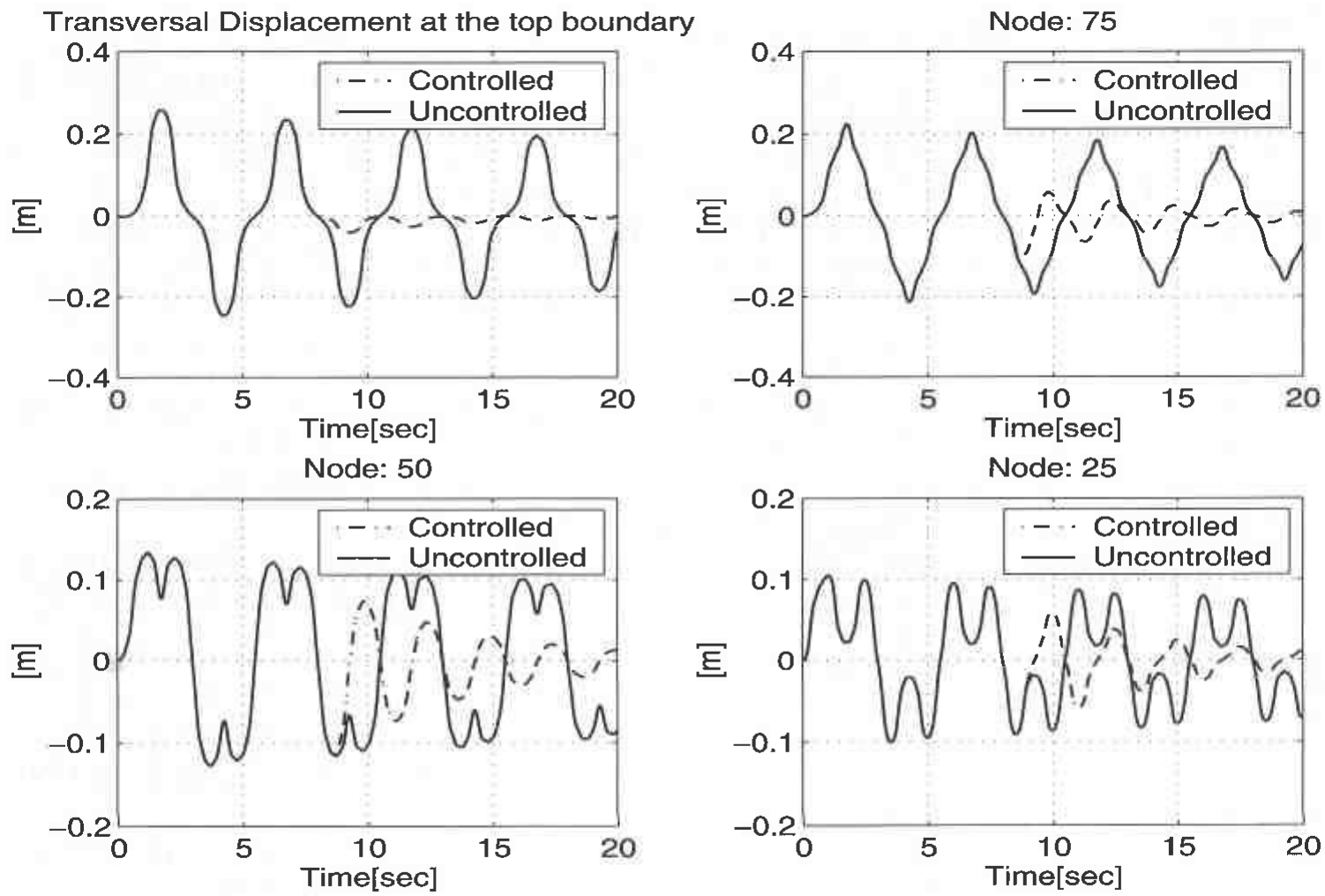

Figure 11. Comparison between controlled and uncontrolled responses of the riser, when several modes of vibration are excited.



Figure 12. Comparison between control forces. 


\section{References}

AAMO, O. M. and Fossen, T. I. (1999). Controlling line tension in thruster assisted mooring systems, in Proc. 1999 IEEE Conf. on Control Applications, Hawaii, 22-26.

Ames, W. F. (1965). Nonlinear Partial Equations in Engineering, Academic Press.

KHaliL, H. K. (1996). Nonlinear Systems, Sec. Ed., Prentic Hall, New York.

Matsuno K. and Murata, K. (1999). Passivity and PDS control of flesible mechanical systems on the basis of distributed parametersystems, in Proc. 1999 IEEE Int. Conf. on Systems, Man, and Cybernetics, Tokyo, Japan, 51-56.

Mrtchell, A. R. and GriffThs, D. F. (1980). The Finite Difference Method in Partial Differential Equations, John Wiley \& Sons, UK.

Richtmyer, R. D. and Mortonon, K. W. (1967). Difference Methods for Initial-Value Problems, Sec. ed., John Wiley \& Sons, New York.

Sмiтн, G. D. (1978). Numerical Solution of Partial Differential Equations: Finite Difference Methods, Sec. ed., Clarendon Press, Oxford, UK.

VAN DER SCHAFT, A. J. (1996). $L_{2}$-Gain and Passivity Techniques in Nonlinear Control, in Lecture Notes in Control and Information Sciences, Springer Verlag, Heidberg, vol. 218.

\section{Appendix}

Lemma 1: Let $\eta(x, t)$ and $\mu(x, t)$ be the solutions of equations of motion which satisfy the corresponding boundary conditions. Then

$$
\begin{aligned}
& -E I \int_{0}^{L}\left[\eta_{x x x x} \eta_{t}-\eta_{x x} \eta_{x x t}\right] d x=-E I\left[\left.\eta_{x x x} \eta_{t}\right|_{0} ^{L}-\left.\eta_{x x} \eta_{t x}\right|_{0} ^{L}\right] \\
& \frac{E A}{2} \int_{0}^{L}\left[\eta_{x}^{3} \eta_{x t}+3 \eta_{x}^{2} \eta_{x x} \eta_{t}\right] d x=\left.\frac{E A}{2} \eta_{x}^{3} \eta_{t}\right|_{0} ^{L} \\
& P_{0} \int_{0}^{L}\left[\eta_{x x} \eta_{t}+\eta_{x} \eta_{x t}\right] d x=\left.P_{0} \eta_{x} \eta_{t}\right|_{0} ^{L} \\
& -\gamma E I \int_{0}^{L} x \eta_{x x x x} \eta_{x} d x=-\gamma \frac{3 E I}{2} \int_{0}^{L} \eta_{x x}^{2} d x-\gamma E I\left[L \left(\eta_{x x x}(L, t) \eta_{x}(L, t)\right.\right. \\
& \left.\left.-\frac{1}{2} \eta_{x x}^{2}(L, t)\right)-\left.\eta_{x x} \eta_{x}\right|_{0} ^{L}\right] \\
& \gamma P_{0} \int_{0}^{L} x \eta_{x x} \eta_{x} d x=\gamma P_{0}\left[\frac{L}{2} \eta_{x}^{2}(L, t)-\frac{1}{2} \int_{0}^{L} \eta_{x}^{2} d x\right] \\
& \gamma \frac{3}{2} E A \int_{0}^{L} x \eta_{x}^{3} \eta_{x x} d x=\gamma_{2}^{\frac{3}{2}} E A\left[\frac{L}{4} \eta_{x}^{4}(L, t)-\frac{1}{4} \int_{0}^{L} \eta_{x}^{4} d x\right] \\
& \gamma \rho A \int_{0}^{L} x \eta_{t} \eta_{t x} d x=\gamma \rho A\left[\frac{L}{2} \eta_{t}^{2}(L, t)-\frac{1}{2} \int_{0}^{L} \eta_{t}^{2} d x\right] \\
& E A \int_{0}^{L}\left(\mu_{x} \mu_{x t}+\mu_{x x} \mu_{t}\right) d x=\left.E A \mu_{x} \mu_{t}\right|_{0} ^{L}
\end{aligned}
$$




$$
\begin{array}{r}
\gamma \rho A \int_{0}^{L} x \mu_{t} \mu_{x t} d x=\gamma \rho A\left[\frac{L}{2} \mu_{t}^{2}(L, t)-\frac{1}{2} \int_{0}^{L} \mu_{t}^{2} d x\right] \\
\gamma E A \int_{0}^{L} x \mu_{x x} \mu_{x} d x=\gamma E A\left[\frac{L}{2} \mu_{x}^{2}(L, t)-\frac{1}{2} \int_{0}^{L} \mu_{x}^{2} d x\right] \\
E A \int_{0}^{L} \eta_{x x} \mu_{x} \eta_{t} d x+E A \int_{0}^{L} \eta_{x} \mu_{x x} \eta_{t} d x+E A \int_{0}^{L} \eta_{x} \eta_{x x} \mu_{t} d x \\
+\gamma E A \int_{0}^{L} x \eta_{x}^{2} \mu_{x x} d x+E A \int_{0}^{L} \eta_{x} \eta_{x t} \mu_{x} d x+\frac{E A}{2} \int_{0}^{L} \mu_{x}^{2} \mu_{x t} d x \\
+2 \gamma E A \int_{0}^{L} x \eta_{x x} \mu_{x} \eta_{x} d x=\left.E A \eta_{x} \mu_{x} \eta_{t}\right|_{0} ^{L}+\gamma E A L \eta_{x}^{2}(L, t) \mu_{x}(L, t) \\
+\left.E A \frac{1}{2} \eta_{x}^{2} \mu_{t}\right|_{0} ^{L}-\gamma E A \int_{0}^{L} \eta_{x}^{2} \mu_{x} d x
\end{array}
$$

apply for all $t \geqslant 0$.

Proof: The proof is straight forward by applying integration by parts and will not be given here. 